\title{
An analytical perspective on the venous return controversy
}

\author{
Valeria María del Rosario Galetti, Eng
}

Received: 26 January 2018/Revised: 5 February 2018/Accepted: 16 May 2018/Published online: 7 June 2018

(c) Canadian Anesthesiologists' Society 2018

\section{To the Editor,}

The recent review by Gelman and Bigatello ${ }^{1}$ follows a model of the systemic circulation that asserts that steadystate cardiac output is driven by the pressure gradient in Equation 1, and the role of the pump (i.e., the heart) is to "accommodate and eject the aliquot of blood received from the venous circulation" 1 when that "aliquot of blood" is, in fact, the heart's output (i.e., steady-state cardiac output).

$Q=\frac{p_{1}-p_{2}}{\Omega}$

(Equation 1)

where:

$p_{1}=$ pressure head located in the peripheral venous system (the ratio of total blood volume and vascular compliance - i.e., mean circulatory filling pressure ${ }^{1}$;

$p_{1}=$ pressure at the end of the systemic circuit - i.e., right atrial pressure;

$Q=$ flow - i.e., venous return or cardiac output (which can be used interchangeably);

$\Omega=$ the apparent resistance across the gradient - i.e., resistance to venous return.

The expression in Equation 1, however, is based on certain misconceptions of Guyton's venous return curves ${ }^{2,3}$ that indicate $p_{1}-p_{2}$ is the source of mechanical energy for blood propulsion. Therefore, this assertion is confronted with a mechanical energy balance (MEB) (Equation 2) ${ }^{4}$

This letter is accompanied by a reply. Please see Can J Anesth 2018; 65: this issue.

\section{M. R. Galetti, Eng ( $ه)$}

Department of Chemical Engineering, National University of Technology of Rosario, Rosario, Argentina

e-mail: valeria.m.galetti@gmail.com

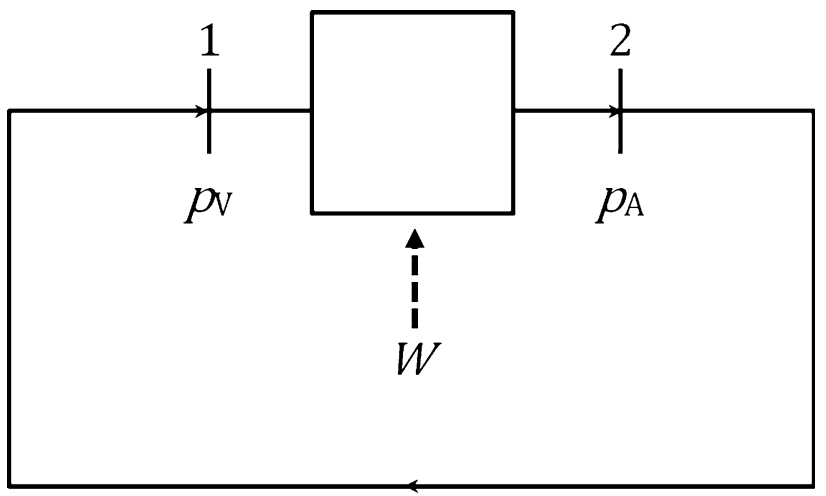

Figure Simplified block diagram of the closed loop of the cardiovascular circuit. $p_{V}=$ venous pressure (at location "1"); $p_{A}=$ arterial pressure (at location “ 2 ")

analysis for isothermal flow of a fluid with constant physical properties, in steady-state condition, through a tubular system with an impulsion pump, with its input port at $(a)$ and its output port at $(b)$. All the terms are in units of energy per unit of mass.

$$
\frac{p_{a}}{\rho}+g z_{a}+\frac{v_{a}^{2}}{2}+W_{p}=\frac{p_{b}}{\rho}+g z_{b}+\frac{v_{b}^{2}}{2}+h_{f}
$$

$$
\text { (Equation 2) }
$$

where

$\frac{p}{\rho}=$ pressure energy term, where $p=$ pressure and $\rho=$ density;

$g z=$ gravitational potential energy term, where $g=$ gravitational acceleration and $z=$ height over reference plane;

$\frac{v^{2}}{2}=$ kinetic energy term, where $v=$ average speed;

$W_{p}$ : mechanical work done on the fluid by the pump; $h_{f}=$ head loss; with: $h_{f}=\frac{f L v^{2}}{2 D}$, where: $f=$ friction factor; $L=$ total length; and 


$$
D=\text { diameter. }
$$

For the "heart" subsystem, we can assume no variation in $z$; negligible variation in $v, f$, and $L$; and with $p$ at input $\left(\right.$ arterial $; p_{A}$ ) and output (venous; $p_{V}$ ) positions. The MEB for the pump subsystem is then:

$$
\begin{aligned}
& \frac{p_{A}-p_{V}}{\rho}=W_{p} \\
& \text { (Equation 3) }
\end{aligned}
$$

Moreover, the only structure doing work $(W)$ in the cardiovascular system in the steady-state condition is, by necessity, the contracting cardiac chambers because work is defined as:

$$
W=\int_{V_{1}}^{V_{2}} p d V
$$

(Equation 4)

where:

$$
d V=\text { differential of volume } .
$$
$V^{5}$

Hence, energy release requires a downward variation in

Conversely, for the "vascular" subsystem, $L$ (and therefore $f$ ) is not negligible. Because it behaves like a passive conduit in the steady-state condition $\left(W_{p}=0\right)$, the MEB for this subsystem (Equation 2) is:

$$
h_{f}=\frac{p_{A}-p_{V}}{\rho}
$$

(Equation 5)

Note that the pressure drop $(\Delta p)$ in the vascular system is the consequence of the dissipation of fluid energy due to hydraulic viscous resistance. In addition, for laminar flow, $h_{f}$ is proportional to the law of Hagen-Poiseuille:

$$
h_{f}=\frac{8 \mu L Q}{\pi R^{4}}
$$

(Equation 6)

where

$Q=$ volumetric flow;

$R=$ vessel radius;

$\mu=$ blood viscosity.

Finally, combining Equations 2, 3, 5, and 6, yields:
$W_{p}=\frac{p_{A}-p_{V}}{\rho}=h_{f}=\frac{8 \mu L Q}{\pi R^{4}}$

(Equation 7)

This means that as blood flows through the peripheral circulation, an irreversible energy loss due to viscous friction is generated - manifesting as a pressure gradient, or "profile" - which is constantly supplied by the systolic work during each cycle, as shown in Equation 7.

In summary, a similarity is observed between Equations 1, 5, and 6 in that they are limited to the isolated vascular subsystem. An integrative analysis of the cardiovascular system cannot exclude the work supplied by the heart - which Gelman and Bigatello's emphasis on venous return ambiguously does - because, as indicated in Equation 7, it is implicit in the pressure drop $(\Delta p)$ in the vasculature.

Acknowledgement To the memory of Jorge E. Robin.

Conflicts of interest None declared.

Editorial responsibility This submission was handled by Dr. Hilary P. Grocott, Editor-in-Chief, Canadian Journal of Anesthesia.

\section{References}

1. Gelman $S$, Bigatello $L$. The physiologic basis for goal-directed hemodynamic and fluid therapy: the pivotal role of the venous circulation. Can J Anesth 2018. DOI: https://doi.org/10.1007/ s12630-017-1045-3.

2. Brengelmann GL. A critical analysis of the view that right atrial pressure determines venous return. J Appl Physiol 1985; 2003(94): 849-59.

3. Beard DA, Feigl EO. Understanding Guyton's venous return curves. Am J Physiol Heart Circ Physiol 2011; 301: H629-33.

4. McCabe WL, Smith JC, Harriott P. Unit equations in flow of fluids. In: Warren L, Smith JC, McCabe H, editors. Unit Operations of Chemical Engineering. 4th ed. NY: McGraw-Hill; 1993. p. 78.

5. Brengelmann GL. Point-counterpoint: Rebuttal from Dr. Brengelmann. J Appl Physiol 2006; 101: 1527. 